\title{
Million-atom molecular dynamics simulation by order-N electronic structure theory and parallel computation
}

\author{
Masaaki Geshi, Takeo Hoshi and Takeo Fujiwara \\ Department of Applied Physics, University of Tokyo, \\ Hongo 7-3-1, Bunkyo-ku, Tokyo 113-8656
}

(Received February 7, 2020)

\begin{abstract}
Parallelism Of Tight-Binding Molecular Dynamics Simulations Is Presented By Means Of The Order-N Electronic Structure Theory With The Wannier States, Recently Developed (J. Phys. Soc. Jpn. 69,3773 (2000)). An Application Is Tested For Silicon Nanocrystals Of More Than Millions Atoms With The Transferable Tight-Binding Hamiltonian. The Efficiency Of Parallelism Is Perfect, 98.8\%, And The Method Is The Most Suitable To Parallel Computation. The Elapse Time For A System Of $2 \times 10^{6}$ Atoms Is 3.0 Minutes By A Computer System Of 64 Processors Of Sgi Origin 3800. The Calculated Results Are In Good Agreement With The Results Of The Exact Diagonalization, With An Error Of $2 \%$ For The Lattice Constant And Errors Less Than 10\% For Elastic Constants.
\end{abstract}

KEYWORDS: order- $\mathrm{N}$ method, parallel computation, tight-binding Hamiltonian, silicon nanocrystal

\section{Introduction}

Accurate large-scale atomistic simulations are very important to investigate and to predict various properties of materials. For this purpose, the first principle electronic structure theories have been extended to calculations of the total energy and forces and the first principle molecular dynamics (MD) simulation or the CarParrinello method ${ }^{1)}$ are now used quite widely in the condensed matter physics. However, the systems for the first principle MD simulations are practically limited to much smaller size, at most, of hundreds atoms and much shorter time period of few tens pico-seconds. The other extreme is the classical MD simulations with short-range interatomic potentials which are applied to systems of millions or ten millions atoms with time period of a few hundreds pico-seconds. ${ }^{2,3)}$ Classical MD simulations are very useful to investigate nanoscale systems when accurate interatomic potentials can be used. Even so, applicability of classical MD simulations is limited to phenomena in which electronic process does not play an essential role.

Modern material technology is deeply involved in electronic processes. Then intense attention has been paid to the order- $\mathrm{N}$ method for the electronic structure calculations, whose computational cost increases in linearly proportion to the number of electrons. ${ }^{4,5)}$

Novel order-N method is being developed on the basis of the Wannier states. ${ }^{6,7)}$ The Wannier states is formally defined with the unitary transformation of the occupied eigen states. Once we get true Wannier states $\left|\psi_{j}\right\rangle$, the density matrix can be defined as

$$
\hat{\rho}=\sum_{j}^{o c c}\left|\psi_{j}\right\rangle\left\langle\psi_{j}\right| .
$$

The expectation value of any physical quantity $X$ can be obtained with the density matrix or the Wannier states as

$$
\langle\hat{X}\rangle=\operatorname{Tr}[\hat{\rho} \hat{X}]=\sum_{j}^{o c c}\left\langle\psi_{j}|\hat{X}| \psi_{j}\right\rangle .
$$

If we put the localization constraint to construct approximate Wannier states with a loss of certain amount of accuracy, then we can formulate the order- $\mathrm{N}$ method and reduce computational cost extremely. Using this order-N method, a system of $1.4 \times 10^{6}$ atoms was calculated by a single CPU standard workstation. ${ }^{8)}$

In the present paper we do parallel computation of the perturbation procedure of the order- $\mathrm{N}$ method, we call the perturbative order-N method. Silicon nanocrystals are calculated up to a system of 2,097,152 atoms, using SGI Origin 3800 system, and the efficiency of parallelism is analyzed. To test an accuracy and applicability for calculation of physical quantities, the lattice constant and elastic constants are calculated using cluster systems of up to 1,423,909 atoms. The usefulness and the limit of the perturbative order- $\mathrm{N}$ method will be discussed in detail.

\section{Theoretical backgrounds}

\subsection{The Wannier states}

The Wannier states centered on the $j$-bond can be expressed as

$$
\left|\psi_{j}\right\rangle=C_{j}^{(0)}\left|b_{j}\right\rangle+\sum_{i(\neq j)} C_{j}^{(i)}\left|a_{i}\right\rangle,
$$

where $C_{j}^{(0)}$ is the mixing coefficient of the central bonding orbital $\left|b_{j}\right\rangle$ and $C_{j}^{(i)}$ is that of the anti-bonding orbital $\left|a_{i}\right\rangle$ on the neighboring $i$-bond. ${ }^{6,7)}$ The mixing of the bonding orbitals on the neighboring bonds are negligibly small due to the orthogonality and the completeness, because they contribute to other Wannier states.

For diamond structure crystals, we adopt the transferable Hamiltonian $\hat{H}$ of Kwon et al. ${ }^{9)}$ The Hamil- 
tonian includes the tight-binding interactions and the short-range repulsive interactions between ion cores. We truncate the hopping interactions between the first and the second neighbor distances. If we denote $\mathrm{sp}^{3}$ hybridized orbitals $\left|h_{i}\right\rangle$, the bonding orbital $\left|b_{j}\right\rangle$ and the anti-bonding orbital $\left|a_{j}\right\rangle$ are linear combinations of the two hybridized orbitals $\left(\left|h_{i}\right\rangle \pm\left|h_{i^{\prime}}\right\rangle\right) / \sqrt{2}$.

In the case of silicon crystals, the exact results for a system of 512 atoms are $\left|C_{j}^{(0)}\right|^{2}=0.938$ and $\sum_{i}\left|C_{j}^{(i)}\right|^{2}$ up to the second bond-steps is 0.995. On the other hand, by the first-order perturbation theory the coefficients can be given by an equation

$$
\frac{C^{(\nu(i))}}{C^{(0)}}=\frac{\left\langle a^{(\nu(i))}|\hat{H}| b_{k}\right\rangle}{\varepsilon_{b}-\varepsilon_{a}},
$$

and this gives $\left|C_{j}^{(0)}\right|^{2}=0.934 .^{6,7)}$ Note that, the firstorder perturbation theory gives the value 1 for $\sum_{i}\left|C_{j}^{(i)}\right|^{2}$ up to the second bond-steps.

\subsection{Perturbative order- $N$ method}

The total energy in the tight-binding formalism is given as

$$
E_{\mathrm{tot}}=E_{\mathrm{bs}}+E_{\mathrm{rep}},
$$

where $E_{\mathrm{bs}}$ is the band structure (BS) energy and $E_{\mathrm{rep}}$ is the repulsive energy. On the basis of the Wannier states $\left|\psi_{j}\right\rangle$, the BS energy and its contribution to forces on the $I$ atom (site $\mathbf{R}_{I}$ ) are written, with the tight-binding Hamiltonian $\hat{H}$, as ${ }^{6)}$

$$
E_{\mathrm{bs}} \equiv \operatorname{Tr}[\hat{\rho} \hat{H}]=\sum_{j}^{o c c}\left\langle\psi_{j}|\hat{H}| \psi_{j}\right\rangle
$$

and

$$
\mathbf{F}_{I}^{\mathrm{bs}} \equiv \operatorname{Tr}\left[\hat{\rho} \frac{\partial \hat{H}}{\partial \mathbf{R}_{I}}\right]=-\sum_{j}^{o c c}\left\langle\psi_{j}\left|\frac{\partial \hat{H}}{\partial \mathbf{R}_{I}}\right| \psi_{j}\right\rangle
$$

We will calculate the Wannier states by using the perturbative treatment Eqs. (3) and (4) and the density matrix should be given in the same equation as Eq. (1) with calculated Wannier states $\left|\psi_{j}\right\rangle$. The computational cost of the procedure is linearly scales by the number of electrons $N,{ }^{6,7)}$ and this procedure we call the perturbative order-N method.

When we use the variational procedure to obtain the Wannier states, the physical quantities should be calculated in a way consistent with the calculation of the Wannier states. ${ }^{6,7)}$ Therefore, the density matrix in the above Eqs. (6) and (7) should be replaced by the optimal one $\tilde{\rho}=2 \hat{\rho}-\hat{\rho}^{2}$, and this procedure we call the variational order-N method.

\subsection{Linearly scaling property of perturbative order- $N$ method}

The Wannier states and the matrix elements of the Hamiltonian are given on the basis of the atomic orbitals $\left|\phi_{I \alpha}\right\rangle$. Then we can estimate the computational cost in the following way. Firstly the above physical quantities can be rewritten, by using the matrix elements of the
Hamiltonian and the density matrix represented by the atomic orbitals, as

$$
\begin{array}{r}
E_{\mathrm{bs}}=\sum_{I}^{N_{\text {atom }}} \sum_{\Delta}^{N_{\text {loc }}} \sum_{\alpha}^{N_{\nu}} \sum_{\beta}^{N_{\nu}} \rho_{I \alpha(I+\Delta) \beta} \\
\times\left\langle\phi_{I \alpha}|\hat{H}| \phi_{(I+\Delta) \beta}\right\rangle,
\end{array}
$$

and

$$
\begin{array}{r}
\mathbf{F}_{I}^{\mathrm{bs}}=-\sum_{J}^{N_{\text {atom }}} \sum_{\Delta}^{N_{\text {loc }}} \sum_{\alpha}^{N_{\nu}} \sum_{\beta}^{N_{\nu}} \rho_{J \alpha(J+\Delta) \beta} \\
\times\left\langle\phi_{J \alpha}\left|\frac{\partial \hat{H}}{\partial \mathbf{R}_{I}}\right| \phi_{(J+\Delta) \beta}\right\rangle .
\end{array}
$$

The numbers $N_{\text {atom, }}$ and $N_{\nu}$ are those of atoms and atomic orbitals per atom, respectively. The number $N_{\text {loc }}$ is that of interacting atoms in the local region around the central atom. The local region is defined, outside which the matrix elements of the tight-binding Hamiltonian vanish. In the diamond structure, $N_{\text {loc }}=17$ including the central, the first and the second neighbor atoms.

From Eqs. (8) and (9), the total computation time of the matrix elements are scaled by a factor $N_{\nu} \times N_{\nu} \times$ $N_{\text {loc }} \times N_{\text {atom }}$, where the factor $N_{\nu} \times N_{\nu}$ is due to the cost for the quantum mechanical calculation. In the $\mathrm{sp}^{3}$ minimal basis set, $N_{\nu}$ is four. Therefore, we can calculate each Wannier state by a local procedure and the total computation cost is proportional to the number $N_{\text {atom }}$. The procedure is then the perfect order- $\mathrm{N}$ method.

Non-negligible amount of computation time is consumed in the calculation of the repulsive energy and forces. The part of the listing of the neighboring atoms is also important and its cost is not negligible for large systems. The above two parts are the same as in standard classical MD simulations and the computation time of these two parts are scaled by a factor $N_{\text {loc }} \times N_{\text {atom. }}$ The computation time of the BS energy and forces costs, at least, $N_{\nu} \times N_{\nu}$ times more than those of the calculation of repulsive interactions and the listing of the neighboring atoms.

\subsection{Allotment of Wannier states to processors, memory size and communication of data}

Since the perturbative treatment is completely independent among the Wannier states, we can parallelize the computation with respect to several groups of the Wannier states. When we use $N_{\mathrm{CPU}}$ processors, each processor participates in the calculation of about $N / N_{\mathrm{CPU}}$ states among the total $N$ Wannier states. For example, the calculation of $\left\{\left\langle\psi_{j}|\hat{H}| \psi_{j}\right\rangle\right.$ and $\left\langle\psi_{j}\left|\frac{\partial \hat{H}}{\partial \mathbf{R}_{I}}\right| \psi_{j}\right\rangle$ \}$_{j=j_{n-1}+1, \cdots, j_{n}},\left(j_{n}-j_{n-1} \simeq N / N_{\mathrm{CPU}}\right)$ is allotted to the $n$-th processor.

The matrix elements are not stored on memory because they require totally a large CPU memory. For example, the total memory size for the matrix elements $\left\{\left\langle\phi_{I \alpha}|\hat{H}| \phi_{(I+\Delta) \beta}\right\rangle\right\}$ can be estimated to be $8(\mathrm{~B}) \times 4^{2} \times$ $17 \times 10^{6}=2.2(\mathrm{~GB})$ for a system of $10^{6}$ atoms. Therefore, we calculate the matrix elements when they are required and do not store them. 


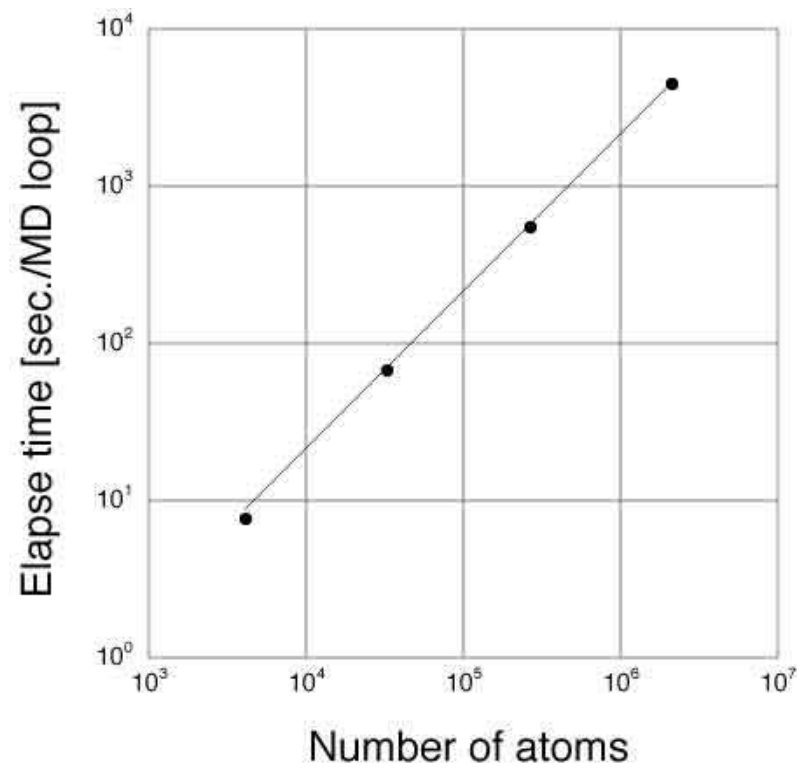

Fig. 1. The elapse time of one MD loop by using one processor for the systems of 4,096, 32,768, 262,144 and 2,097,152 atoms of $\mathrm{Si}$ crystals.

In the case of $10^{6}$ atoms, the memory size for all atomic positions is $8(\mathrm{~B}) \times 3 \times 10^{6}=24(\mathrm{MB})$, that for the listing of the neighboring atoms is $4(\mathrm{~B}) \times 16 \times 10^{6}=64(\mathrm{MB})$, and that for the force is $8(\mathrm{~B}) \times 3 \times 10^{6}=24(\mathrm{MB})$. Then the total memory size for each processor is not large. In the present calculation, all processors have the same data of all atomic positions and no data are communicated among processors during the calculation of the Wannier states and contributions to the BS energy and forces.

After calculating contributions to the BS energy and forces in individual processor, we should sum up these elements. This procedure is accomplished by MPI_ALLREDUCE command in the Message Passing Interface (MPI) in the present calculation. This is wasteful procedure with respect to the communication of the data because a large number of communicated data is not necessary, that is, null. Notwithstanding, the communication time would be relatively cheap expenses in the present work because there exist very heavy computations with respect to quantum mechanical freedoms.

\section{Results and discussions}

\subsection{Elapse time of one processor}

We calculated four different systems of 4,096, 32,768, 262,144 and 2,097,152 atoms of Si crystals with the periodic boundary condition. Figure 1 shows the elapse time of one MD loop and one can see the almost perfect linearly scaling property. The elapse times for the calculation of the BS energy and forces are $97.44 \%$, $97.65 \%, 97.63 \%$ and $97.64 \%$ of the total elapse times in respective systems, which are larger than a factor $16 /(16+1)=0.941$, estimated simply in the case of $N_{\nu} \times N_{\nu}=16$. Note that the small difference between two numbers 0.976 and 0.94 is very serious for the speedup ratio discussed later. From these results, we can conclude that the tight-binding calculation is heavier by a

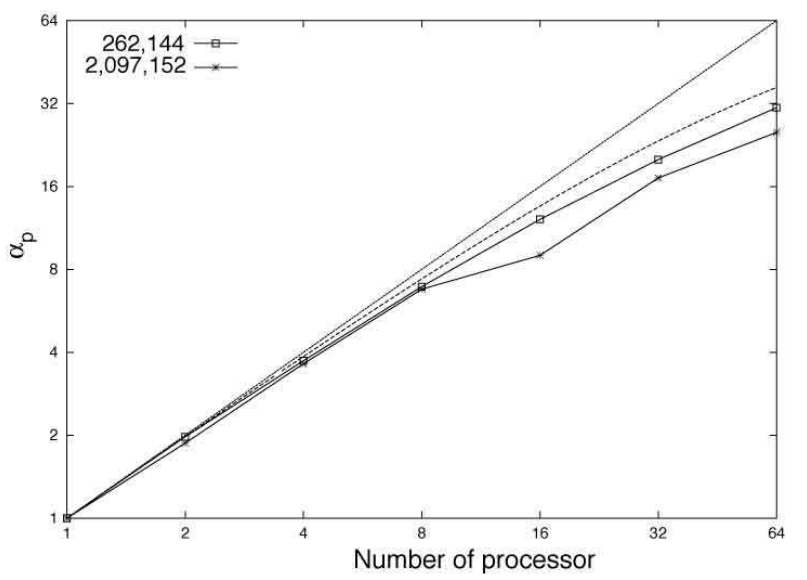

Fig. 2. The speed-up ratio $\alpha_{p}$ for systems of 262,144 and $2,097,152$ atoms as a function of the number of processors. That for a system of 32,768 atoms locates slightly above that of 262,144 atoms. The dashed lines are the maximum speed-up ratio $\left(\alpha_{p}\right)_{\max }$ of $P=0.988$ and dotted lines are just $\left(\alpha_{p}\right)_{\max }=N_{\mathrm{CPU}}$ corresponding to $P=1$. The observed deviation of the line of $2,097,152$ atoms at 16 processors is due to the consumption of the elapse time in the data communication, presumably because of an ill balancing of the data size and the number of processors.

factor $2.5\left(=\frac{0.976}{1-0.976} \times \frac{17-16}{16}\right)$ than our naive estimation. This is very crucial difference in actual simulations, and quantum mechanical calculations are much heavier than the classical simulations even in the tight-binding calculation. One should parallelize firstly the part of the calculation of the BS energy and forces. In practice, we parallelize also the part of the calculation of the repulsive interaction energy. The parallelizable fraction $P$, the fraction of the elapse time of strictly parallelizable part among the whole elapse time in the case of one processor, reaches to 0.988 . This high value of $P$ would be impossible without the generic property of the linear scaling of the present method. Therefore, the perturbative order$\mathrm{N}$ method is one of the most suitable procedures to the parallel computation.

\subsection{Speed-up ratio by parallelism}

When we parallelize the computational program by using $N_{\mathrm{CPU}}$ processors, the speed-up ratio $\alpha_{p}$ is defined as the ratio of the elapse time $t_{N_{\mathrm{CPU}}}$ of $N_{\mathrm{CPU}}$ processors and that $t_{1}$ of one processor as

$$
\alpha_{p} \equiv \frac{t_{1}}{t_{N_{\mathrm{CPU}}}} .
$$

Let us assume that we can parallelize the part of the fraction $P$ perfectly. In other words, we assume that the elapse time of this part can be reduced by a factor $1 / N_{\mathrm{CPU}}$. In such strictly parallelized case, the total elapse time can be minimized with the maximal speed-up ratio $^{10)}$

$$
\left(\alpha_{p}\right)_{\max }=t_{1} \times\left(\frac{1}{t_{N_{\mathrm{CPU}}}}\right)_{\max } \equiv \frac{1}{(1-P)+\frac{P}{N_{\mathrm{CPU}}}} .
$$

The high speed-up ratio is possible only for the high value of the parallelizable fraction $P$. 
(a)

(b)
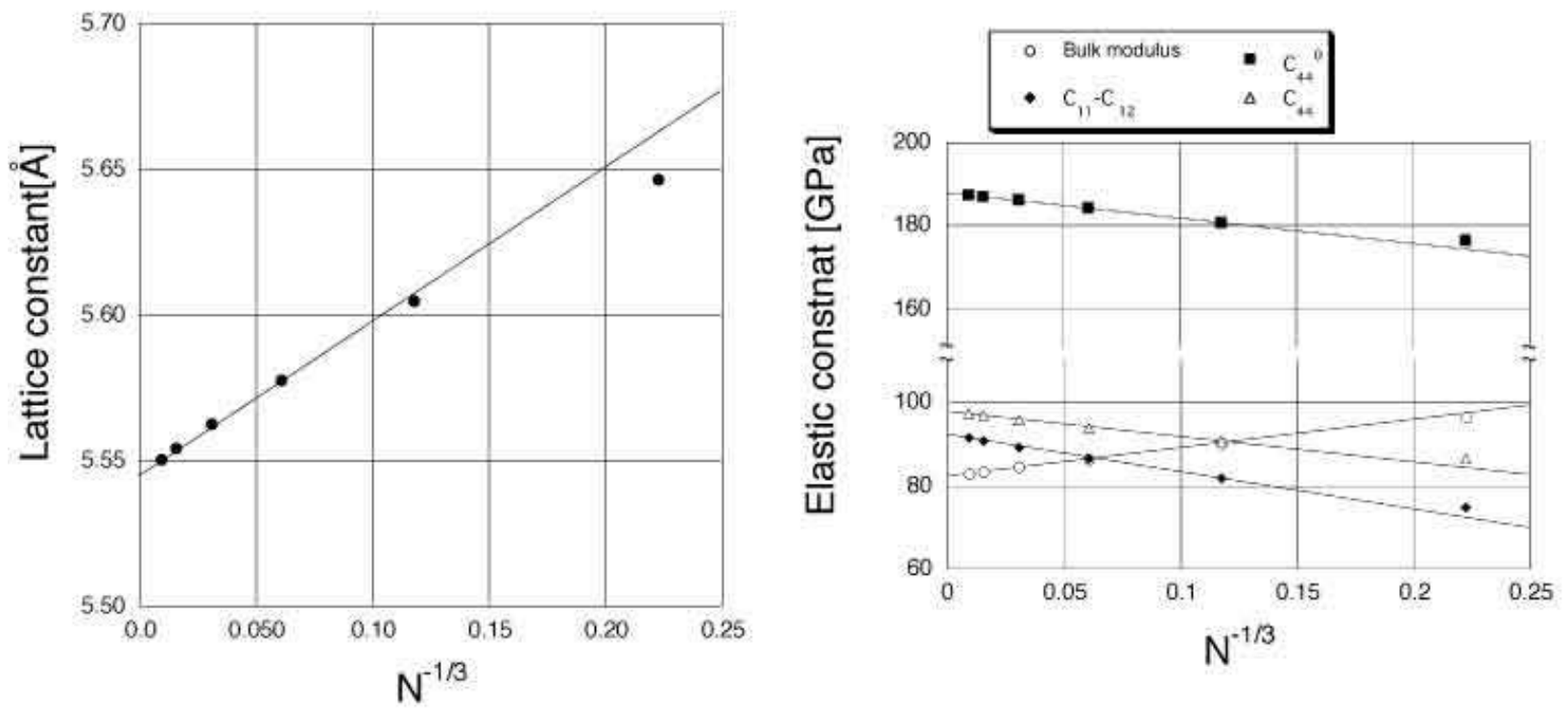

Fig. 3. The lattice constant (a) and elastic constants (b) as a function of $N^{-1 / 3}$, obtained by the perturbative method.

\begin{tabular}{|c|c|c|c|c|}
\hline & Present work & Diag. ${ }^{9)}$ & LDA ${ }^{12)}$ & Exp. \\
\hline Lattice constant $[\AA \bar{A}]$ & $5.546(2.19 \%)$ & 5.427 & 5.431 & $5.429^{13)}$ \\
\hline Bulk modulus [GPa] & $82.3(6.05 \%)$ & 87.6 & 93.0 & $97.8^{14)}$ \\
\hline$C_{11}-C_{12}[\mathrm{GPa}]$ & $92.3(1.70 \%)$ & 93.9 & 98.0 & $101.2^{14)}$ \\
\hline$C_{44}^{0}[\mathrm{GPa}]$ & $188.3(5.14 \%)$ & 198.5 & 111.0 & \\
\hline$C_{44}[\mathrm{GPa}]$ & $97.9(10.0 \%)$ & 89.0 & 85.0 & $79.6^{14)}$ \\
\hline
\end{tabular}

Table I. Lattice constant and elastic constants extrapolated to $N \rightarrow \infty$ systems are compared with those by the diagonalization method (Diag.), the first-principles calculation within the Local Density Approximation (LDA), and experiments (Exp.). The values in parentheses are errors against those with the diagonalization method. The elastic constant $C_{44}^{0}$ is that in a system where the internal displacement is not relaxed.

Figure 2 shows the observed speed-up ratio $\alpha_{p}$ by using MPI and the maximum one $\left(\alpha_{p}\right)_{\max }$ with $P=0.988$ as a function of the number of processors. Compared with the minimum elapse time $t_{1} /\left(\alpha_{p}\right)_{\max }$, the present computation consumes $2 \%$ more for 32,768 atoms ( 8 processors), $18 \%$ more for 262,144 atoms (64 processors), and $45 \%$ more for 2,097,152 atoms (64 processors), respectively. In the case of 64 processors for 2,097,152 atoms, the communication cost is about $30 \%$ among whole elapse time. The cost of data communication increases with the number of the processors. The above analysis indicates that, when one uses a computer system of more than 200 processors for 2,097,152 atoms, the data communication process would consume the majority of the elapse time.

For a system of 2,097,152 atoms, the elapse time of one MD loop by one processor is $4565 \mathrm{~s}$ (76.1 $\mathrm{min})$. When the time interval of one MD step corresponds to 3 femto seconds in physical systems, a one pico second simulation needs 422 hours (17.6 days) of CPU time by one processor. Such simulation becomes feasible when we use 128 processors.

\subsection{Calculations of elastic constants}

The lattice constant and elastic constants are calculated in systems of several sizes of clusters of Si crystal of up to $1,423,909$ atoms. The boundary condition is such that hybridized orbitals of the ideal $\mathrm{sp}^{3}$ type are fixed on the surface atoms but surface atoms can move. Since the elastic constants are the linear response to small distortions, they may be expected to be reproduced by the first order perturbation calculations.

Figure 3 shows the calculated results with increasing the number of atoms $N$. The deviation can be scaled by $N^{-1 / 3}$ because it is the effects of the surface. The values extrapolated to $N \rightarrow \infty$ are summarized in Table I. The present results agree with those of the exact diagonalization method ${ }^{9)}$ within less than $10 \%$ error. The errors except for $C_{44}$ are not more than the difference between results by the present tight-binding Hamiltonian (Diag.) and the LDA. The deviation of the shear modulus $C_{44}$ is larger than those of the bulk modulus or $C_{11}-C_{12}$, since $C_{44}$ is inherently complicated due to the rehybridization and the internal distortion ${ }^{11,12)}$ and this phenomena cannot be described very accurately by the first order perturbation of the Wannier states of fixed $\mathrm{sp}^{3}$ hybrids. The discrepancy between the results with the order- $\mathrm{N}$ method and those with the diagonalization method originates from the localization constraint for constructing the Wannier states and the perturbation treatment in Eqs. (3) and (4). These restrictions are 
controllable and the discrepancy is not serious between the results by the present order- $\mathrm{N}$ method and the diagonalization method. This is a typical example of account balancing between the accuracy and the computational cost in the order- $\mathrm{N}$ method.

Much larger error is found in the value of $C_{44}^{0}$ of the tight-binding calculation itself, compared with that of the LDA calculation, which is a limitation of the present tight-binding Hamiltonian. One of the important works in future is the construction of more accurate tightbinding Hamiltonian from the first principle electronic structure calculations. Even if such sophisticated Hamiltonian is much complicated, it does not cause any essential difficulty in the calculation by the present order- $\mathrm{N}$ method, though it may increase the CPU times.

\section{Conclusion}

In a summary, we demonstrated the efficiency of parallelism of the perturbative order- $\mathrm{N}$ method in the largescale tight-binding MD simulations. The method was shown to be the most profitable procedure for the parallel computation. The communication time is, even in the present case, much less than the time of the quantum mechanical calculations.

The perturbative order- $\mathrm{N}$ method may be hardly applied to systems with large distortion of lattices or bond breaking because the deviation from the unperturbed states becomes very large and, in the bond breaking process, the charge transfer and re-bonding are essentially important. In such cases we should combine the perturbative order-N method with other methods for constructing basis states. The variational method can be associated with the perturbative order- $\mathrm{N}$ method and we can compose a hybrid order- $\mathrm{N}$ method. The hybrid order-N method can give electronic structures in the whole system and, more importantly, there is no discontinuous boundary in the connected region. The hybrid scheme of the perturbative and variational order N-methods has been already applied to the fracture propagation in $\mathrm{Si}$ nanocrystals with $1.4 \times 10^{6}$ atoms without parallelism. ${ }^{8)}$ The parallelized hybrid order- $\mathrm{N}$ method is a very essential method to pursue tight-binding MD simulations for systems of millions atoms and the perturbative order-N method is the key technique in order to enlarge the size of the whole systems.

\section{Acknowledgments}

We are very grateful for useful discussion about general techniques of parallelism by MPI with F. Shimizu and H. Kimizuka. Computation has been done at the Center for Promotion of Computational Science and Engineering (CCSE) of Japan Atomic Energy Research Institute (JAERI) and also partially carried out by use of the facilities of the Supercomputer Center, Institute for Solid State Physics, University of Tokyo. This work is financially supported by Grant-in-Aid from the Ministry of Education, Culture, Sports, Science and Technology and also by "Research and Development for Applying advanced Computational Science and Technology" of Japan Science and Technology Corporation.

1) R. Car and M. Parrinello, Phys. Rev. Lett. 55, 2471 (1985).

2) J. Q. Broughton, F. F. Abraham, N. Bernstein and E. Kaxiras, Phys. Rev. B60, 239 (1999).

3) M. E. Bachlechner, A. Omeltchenko, A. Nakano, R. Kalia, P. Vashishta, I. Ebbsjö, and A. Madhukar, Phys. Rev. Lett. 84, $322(2000)$.

4) S. Goedecker. Rev. Mod. Phys., 71, 1085 (1999).

5) P. Ordejón. Comp. Mat. Sci., 12, 157 (1998).

6) T. Hoshi and T. Fujiwara, J. Phys. Soc. of Jpn. 69, 3773 (2000).

7) T. Hoshi and T. Fujiwara, Surf. Sci. 493, 659 (2001).

8) T. Hoshi and T. Fujiwara, cond-mat/0210366.

9) I. Kwon, R. Biswas, C.Z. Wang, K. M. Ho, and C. M. Soukoulis, Phys. Rev. B 49, 7242 (1994).

10) G. M. Amdahl, AFIPS Conference Proceedings 30 (Atlantic City, N.J., April 18-20), AFIPS Press (1967) pp. 483-485.

11) W. A. Harrison, Electronic structure and the properties of solids (W. H. Freeman and Company, San Fransisco, 1980).

12) O. H. Nielsen and R. M. Martin, Phys. Rev. B 32, 3792 (1985).

13) J. Donohue, The Structure of Elements (Wiley, New York, 1974)

14) H.J. McSkimin, J. Appl. Phys. 24, 988 (1953); H.J. McSkimin and P. Andreatch, ibid. 35, 3312 (1964). 